\title{
QCD phase diagram for finite imaginary chemical potential with HISQ fermions
}

\author{
Jishnu Goswami* \\ Fakultät für Physik, Universität Bielefeld, D-33615 Bielefeld, Germany \\ E-mail:jishnu@physik.uni-bielefeld.de \\ Frithjof Karsch \\ Fakultät für Physik, Universität Bielefeld, D-33615 Bielefeld, Germany \\ Physics Department, Brookhaven National Laboratory, Upton, NY 11973, USA \\ E-mail: karsch@physik.uni-bielefeld.de, karsch@bnl.gov
}

Anirban Lahiri

Fakultät für Physik, Universität Bielefeld, D-33615 Bielefeld, Germany

E-mail: alahiri@physik.uni-bielefeld.de

\section{Christian Schmidt}

Fakultät für Physik, Universität Bielefeld, D-33615 Bielefeld, Germany

E-mail: schmidt@physik.uni-bielefeld.de

\begin{abstract}
We present results from an ongoing study of the phase diagram of (2+1)-flavor QCD using the HISQ action with smaller than physical light quark masses in the Roberge-Weiss (RW) plane on lattices with temporal extent $N_{\tau}=4$. We find that the endpoint of the $1^{\text {st }}$ order RW transition line remains $2^{\text {nd }}$ order at least down to light quark masses corresponding to Goldstone pion masses of $55 \mathrm{MeV}$. Furthermore, we show that the chiral condensate is sensitive to the RW transition and may serve as a energy-like operator characterizing universal behavior close to the RW endpoint.
\end{abstract}

The 36th Annual International Symposium on Lattice Field Theory - LATTICE2018

22-28 July, 2018

Michigan State University, East Lansing, Michigan, USA.

${ }^{*}$ Speaker. 


\section{Introduction}

The exploration of the structure of the phase diagram of strongly interacting matter is an active field of research both in the experimental and theoretical nuclear physics communities. For vanishing values of the chemical potential it is well established that the chiral transition is a crossover at physical values of the two light and the heavier strange quark masses. However, in the limit of vanishing light quark masses the nature of the chiral transition is not yet established undoubtedly. In particular, it is not settled whether the transition is $2^{\text {nd }}$ order or becomes $1^{\text {st }}$ order below a certain critical value of the light quark masses. Earlier work from Pisarski and Wilczek [1] suggests that for two massless flavors $\left(N_{f}=2\right)$ the chiral transition will be $2^{\text {nd }}$ order belonging to the $O(4)$ universality class if the axial $U(1)$ symmetry effectively gets restored only at temperatures higher than that for $S U(2)_{L} \times S U(2)_{R}$ flavor chiral symmetry restoration. If the residual $U_{A}(1)$ breaking is small already at the latter temperature the transition may become $1^{\text {st }}$ order.

Studies with unimproved staggered fermions $[2,3,4]$ show that the chiral transition is $1^{\text {st }}$ order below a certain critical quark mass, which led to a possible version of the phase diagram in the light and strange quark mass plane (Columbia plot) as shown in Fig. 1 (left). However when using improved staggered fermion actions, e.g. HISQ or stout, no $1^{\text {st }}$ order transitions is found even for quite small quark masses, corresponding to Goldstone pion masses as small as $50 \mathrm{MeV}[5,6]$. This may imply that the chiral phase transition in $(2+1)$-flavor QCD is $2^{\text {nd }}$ order in the limit of vanishing light up and down quark masses.

The calculations performed with unimproved staggered fermions $[2,3,4]$ also suggest that the region of $1^{\text {st }}$ order chiral transitions increases in terms of critical quark or pion masses at non-zero imaginary values of the chemical potential (see Fig. 1 (right)), i.e. for negative $\mu^{2}$. Calculations performed at the largest $|i \mu / T|=\pi / 3$ thus can set limits on $m_{\pi}^{c r i}$ in the $\mu=0$ plane. Here we report on our ongoing studies of the QCD phase diagram with imaginary chemical potential and small values of the light quark masses, keeping the strange quark mass at its physical value.
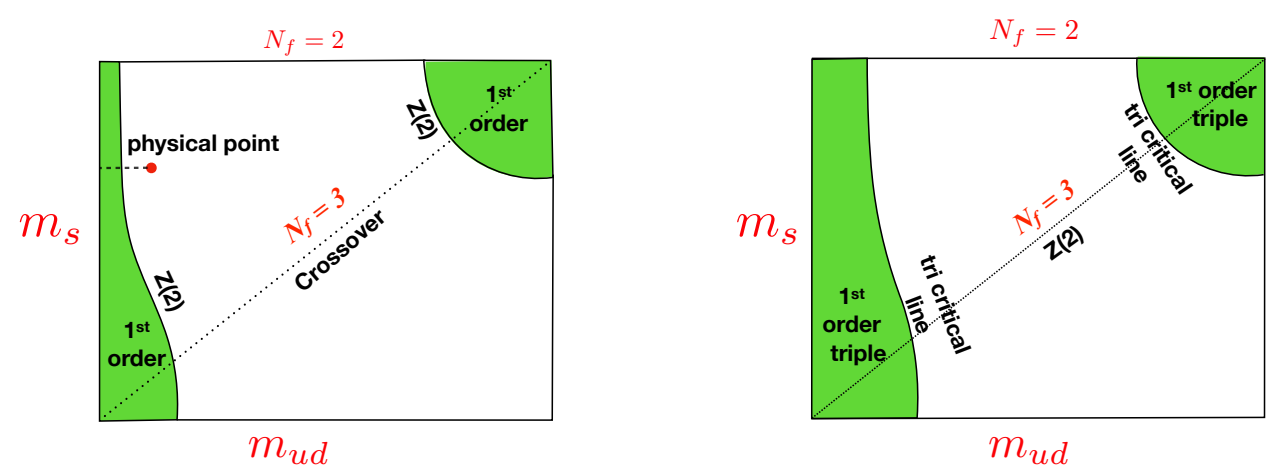

Figure 1: Sketch of possible Coloumbia plots in the $\mu=0$ (left) and $i \mu / T=i \pi / 3$ (right) planes.

\section{Details of the Roberge-Weiss (RW) plane}

At imaginary values of the chemical potential, $i \mu$, the QCD partition function still has a real and positive fermion determinant. As the gauge fields can always be transformed by globally multiplying all time-like gauge field variables $U_{x, \hat{0}}$ with an element of the center of the SU(3) group, 


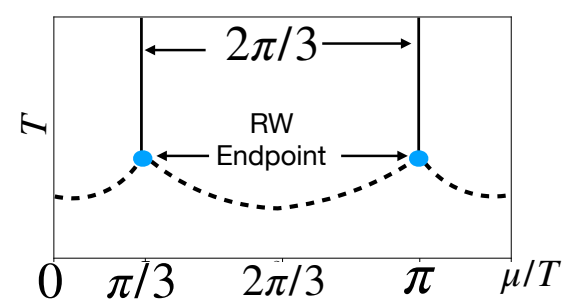

Figure 2: A possible phase diagram in the imaginary chemical potential plane. Solid lines show first order phase transitions, dotted lines correspond to crossover transitions and the blue points indicate $2^{\text {nd }}$ order phase transition points. the partition function is periodic under shifts $\mu / T \rightarrow \mu / T+2 \pi / 3$, i.e. $Z(\mu / T)=Z(\mu / T+$ $2 \pi / 3)$. It has been pointed out by Roberge and Weiss [7] that this leads to a periodicity at $\mu / T=(2 k+1) \pi / 3$ and that a phase transition may occur for these values of the imaginary chemical potential at high temperature. These particular choices of the imaginary chemical potential in a given $\mathrm{Z}(3)$ sector are known as the Roberge-Weiss (RW) planes.

A specific scenario for phase transitions in the RW plane, consistent with the results presented here, is shown in Fig. 2. Alternative scenarios have been found in calculations with standard staggered and also Wilson fermions, where the RW endpoint turns out to be a triple point for sufficiently small values of the light quark masses and three first order transition lines would emerge from this triple point. For $N_{f}=2$ a $1^{\text {st }}$ order triple point has been found on coarse lattices with temporal extent $N_{\tau}=4$ in calculations with standard staggered fermions below $m_{\pi}^{c r i} \sim 400 \mathrm{MeV}$ and with the standard Wilson fermion action below $m_{\pi}^{c r i} \sim 930 \mathrm{MeV}$. The latter critical mass shifted to $m_{\pi}^{c r i} \sim 680 \mathrm{MeV}$ for $N_{\tau}=6$. Results thus are found to be strongly dependent on the fermion discretization scheme and cut-off $\left(N_{\tau}\right)[2,3,4]$. It therfore is not too surprising that these results can change drastically when using improved discretization schemes. In calculations with the stout action and for $N_{f}=2+1$ it is found that for physical pion mass the RW endpoint belongs to the $\mathrm{Z}(2)$ universality class. No $1^{\text {st }}$ order triple point has been found at least for $m_{\pi}>50 \mathrm{MeV}[8,9]$. In calculations using the 2-flavor HISQ action no clear-cut results on the order of the phase transition have been reported so far [10]. In our calculations we use the (2+1)-flavor HISQ action together with an $\mathscr{O}\left(a^{2}\right)$ improved gauge action to examine the nature of the RW-endpoint at smaller than physical values of the light quark masses and physical value of the strange quark mass.

\section{Ising universality and finite size scaling}

If the endpoint of the line of first order transitions in the RW plane is second order, it will belong to the 3-d Z(2) universality class. Near this critical point physics can be described by an effective Ising Hamiltonian, which characterizes the universal critical behavior of any system going through a $Z(2)$ transition. We define

$$
H_{e f f}(t, h)=t \mathscr{E}+h \mathscr{M},
$$

where, $t$ and $h$ are temperature and external field like couplings, that couple to an energy-like operator, $\mathscr{E}$ and a magnetization-like operator $\mathscr{M}$. Under Z(2) transformation, $\mathscr{E} \rightarrow \mathscr{E}$ and $\mathscr{M} \rightarrow$ - $\mathscr{M}$, i.e. $\mathscr{E}$ remains invariant while $\mathscr{M}$ changes sign. Possible choices for these operators are the real and imaginary parts of the Polyakov loop, $\mathscr{E} \sim \operatorname{Re} L, \mathscr{M} \sim \operatorname{Im} L$. However, other choices are possible. For instance, the chiral condensate, $\bar{\psi} \psi$, is invariant under a $\mathrm{Z}(2)$ transformation that changes the imaginary part of all Wilson loops. It thus may equally well serve as the energy-like 
operator in an effective Ising Hamiltonian. We show contour plots of energy-like operators versus the magnetization like operator in Fig. 3. They present the characteristic features of "bananashaped" contours known for Z(2) transitions [11], which is even more obvious for $\bar{\psi} \psi$ than $R e L$.
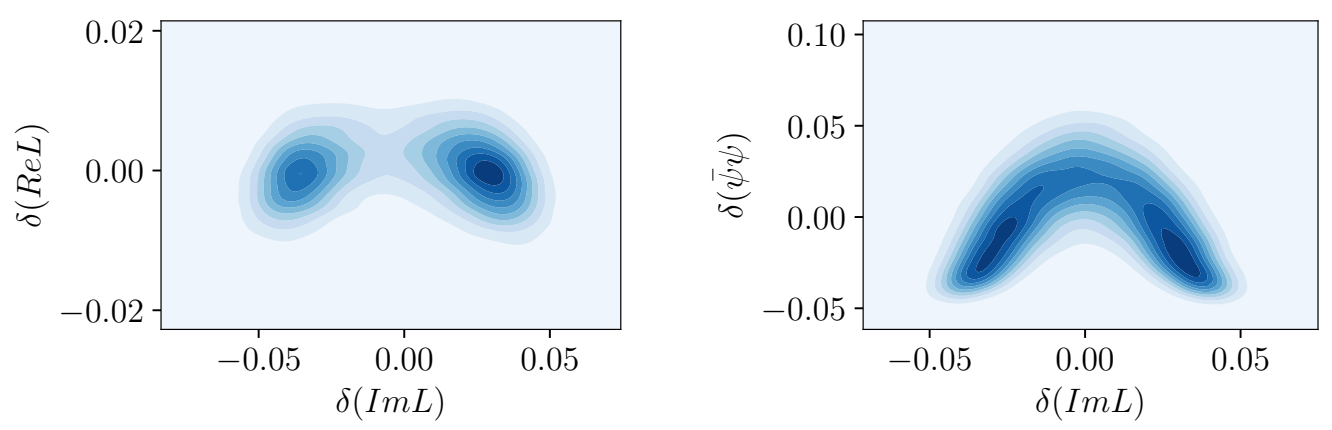

Figure 3: Contour plot of $\operatorname{Re} L$ (left) and $\bar{\psi} \psi$ (right) versus $\operatorname{Im} L$ in the vicinity of the RW endpoint for $m_{l} / m_{s}=1 / 27$.

The logarithm of the QCD partition function defines the free energy density, $f(T, \mu, V)=$ $-\frac{T}{V} \ln Z(T, \mu, V)$, which in the vicinity of a critical point can be split into a singular and regular part,

$$
f \simeq b^{-d} f_{s}\left(b^{1 / v} t / t_{0}, b^{\beta \delta / v} h / h_{0}, b^{-1} N_{\sigma} / l_{0}\right)+f_{r}(T, \mu, V),
$$

where $f_{s}$ is a homogeneous function in terms of the reduced temperature $t=\left(T-T_{c}\right) / T_{c}$, the symmetry breaking field $h=\mu / T-\pi / 3$ and the volume $V=N_{\sigma}^{3}$. They all are expressed in units of non-universal scale parameters $t_{0}, h_{0}$ and $l_{0}$. Eq. 3.2 describes the universal critical behavior close to the critical point $(t, h)=(0,0)$ in the RW plane. From this one may derive the scaling functions for the order parameter $M=-\partial f\left(T, \mu, N_{\sigma}\right) / \partial h$ and its susceptibility $\chi_{h}=-\partial^{2} f\left(T, \mu, N_{\sigma}\right) / \partial h^{2}$. As the symmetry breaking field vanishes in the RW plane, $h=0$, we may set $b=N_{\sigma} / l_{0}$ to obtain the finite size scaling relations for $M$ and $\chi_{h}$,

$$
\begin{aligned}
& M=z_{1} N_{\sigma}^{-\beta / v} f_{G}\left(z_{0} t N_{\sigma}^{1 / v}\right)+\text { reg. }, \\
& \chi_{h}=z_{2} N_{\sigma}^{\gamma / v} f_{\chi}\left(z_{0} t N_{\sigma}^{1 / v}\right)+\text { reg. },
\end{aligned}
$$

with constants $z_{0}, z_{1}$ and $z_{2}$ that are related to the scale parameters $t_{0}, h_{0}$, and $l_{0}$. We also adopted the conventional notation and normalization for the universal scaling functions of the order parameter and the susceptibility, $f_{G}$ and $f_{\chi}$, respectively [12].

\section{Simulation details}

We have performed our calculations at imaginary chemical potential in the RW plane using the HISQ action. This reduces $\mathscr{O}\left(a^{2}\right)$ cut-off effects in the staggered fermion discretization scheme and efficiently suppresses taste-changing interactions. This provides an improved approximation of continuum physics relative to that reached with standard staggered action at the same value of the cut-off. 
The partition function for (2+1)-flavor QCD with two degenerate light quark masses $\left(m_{l}\right)$, a strange quark mass $\left(m_{s}\right)$ and identical chemical potentials $\mu / T=\pi / 3$ for all flavors may be written as,

$$
Z(T, \mu)=\int[\mathscr{D} U] \operatorname{det}\left[M_{l}(i \mu)\right]^{1 / 2} \operatorname{det}\left[M_{s}(i \mu)\right]^{1 / 4} \exp \left[-S_{G}\right],
$$

where, $M_{q}=D_{H I S Q}(i \mu)+m_{q}$. For the gauge action, $S_{G}$, we use the tree level improved Symanzik action. The strange quark mass has been fixed to its physical value and the light quark masses have been varied starting at the physical value, $m_{l} / m_{s}=1 / 27$, towards chiral limit. The smallest value used in our calculations, $m_{l} / m_{s}=1 / 160$, corresponds to a Goldstone pion mass of about $55 \mathrm{MeV}$. Some further details on our simulation parameters are given in Table 1. We vary the temperature

\begin{tabular}{|c|c|c|c|}
\hline$N_{\sigma}$ & $N_{\tau}$ & $m_{l} / m_{s}$ & $m_{\pi}(\mathrm{MeV})$ \\
\hline 8 & 4 & $1 / 27$ & 135 \\
\hline 12 & 4 & $1 / 27$ & 135 \\
\hline 16 & 4 & $1 / 27,1 / 40,1 / 60$ & $135,110,90$ \\
\hline 24 & 4 & $1 / 27,1 / 40,1 / 160$ & $135,110,55$ \\
\hline
\end{tabular}

Table 1: Details of the numerical simulation parameters

in the range, $T \sim T_{c} \pm 0.1 T_{c}$. Generally we generated 20,000 trajectories per $T$ value away from $T_{c}$ and 80,000 trajectories near $T_{c}$.

\section{Results}

\subsection{Scaling in the vicinity of the RW endpoint}

By varying the light quark masses in our simulations we examined the behavior of the order parameter $M$ and the susceptibility $\chi_{h}$ as function of the spatial volume. Even at the smallest quark mass value used in our simulations, $m_{l} / m_{s}=1 / 160$, we find that the peak of the susceptibilities at $T_{c}$ rises significantly slower than the $N_{\sigma}^{3}$ divergence that would be expected for a first order phase transition. We thus compared our results to the expected scaling behavior for a transition in the 3-d, Z(2) universality class. In Fig. 4 (top) we show results for the order parameter $M=\langle|\operatorname{ImL}|\rangle$ (top, left) and the order parameter susceptibility, $\chi_{h}$ (top, right), calculated on lattices with spatial extent $N_{\sigma}=12,16$, and 24 and $m_{l} / m_{s}=1 / 27$. These results for $N_{\sigma}=16$, and 24 have been fitted using the $\mathrm{Z}(2)$ finite size scaling functions. The rescaled data are shown in Fig. 4 (bottom) and include also results for $N_{\sigma}=8$ for comparison. As can be seen for the order parameter possible contributions from regular terms that lead to deviations from the universal $Z(2)$ scaling curve are visible for $N_{\sigma}=8$, but are small. For the susceptibility, however, scaling violations are large in the symmetry broken phase. This also has been observed in simulations of the Ising model and, in addition to contributions from regular terms, may indicate substantial contributions from corrections to scaling [12]. Similar results we find for smaller values of the light quark masses.

\subsection{Chiral observables in the vicinity of the RW endpoint}

As discussed in Section 3 and shown in Fig. 3, the chiral condensate also may serve as an energy-like observable in the vicinity of the RW endpoint. The maximum in the temperature 

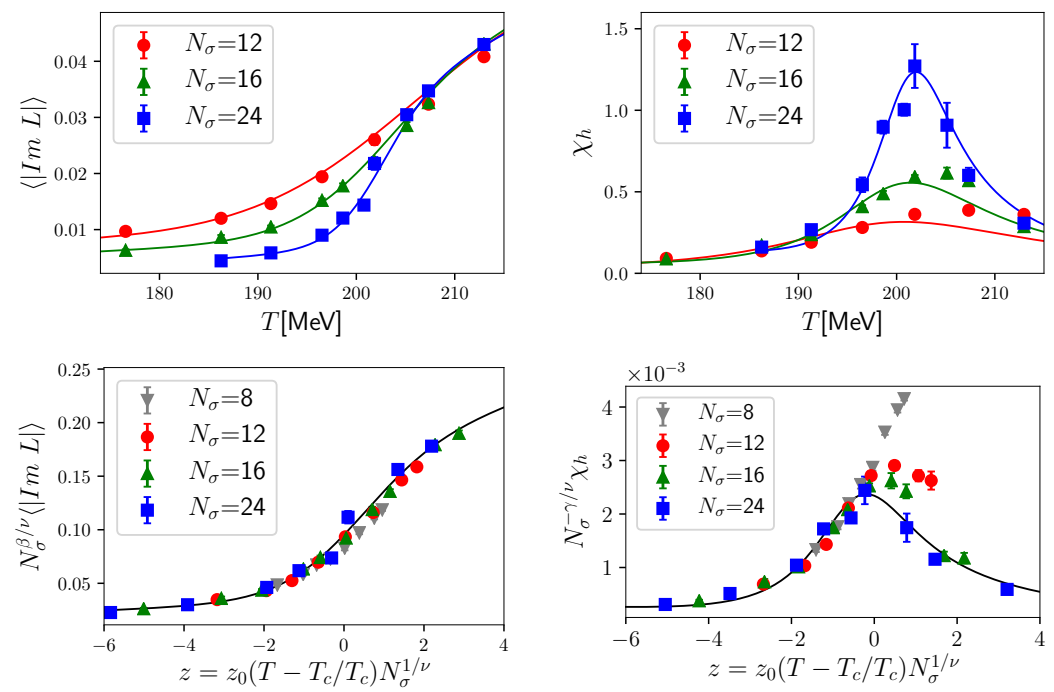

Figure 4: The order parameter $M$ (top, left) and the order parameter susceptibility $\chi_{h}$ (top, right) for $m_{l} / m_{s}=$ $1 / 27$ and various lattice sizes. Lines correspond to fits using the $Z(2)$ finite size scaling functions. The corresponding rescaled data and scaling functions are shown in the figures on the bottom.

derivative of the chiral condensate, the mixed susceptibility, thus may equally well be used to determine the temperature of the RW transition at fixed $m_{l} / m_{s}$. We show in Fig. 5 (left) results for the renormalization group invariant (RGI) chiral condensate

$$
\Delta_{l s}=\frac{m_{s}}{f_{K}^{4}}\left(\langle\bar{\psi} \psi\rangle_{l}-\frac{m_{l}}{m_{s}}\langle\bar{\psi} \psi\rangle_{s}\right)
$$

where we used the kaon decay constant $f_{K}$ for normalization. Its temperature derivative is shown Fig. 5 (right). The quark mass dependence of the peak location as well as the weak dependence of the peak height on the quark mass is found to be in agreement with that of the order parameter susceptibility $\chi_{h}$.
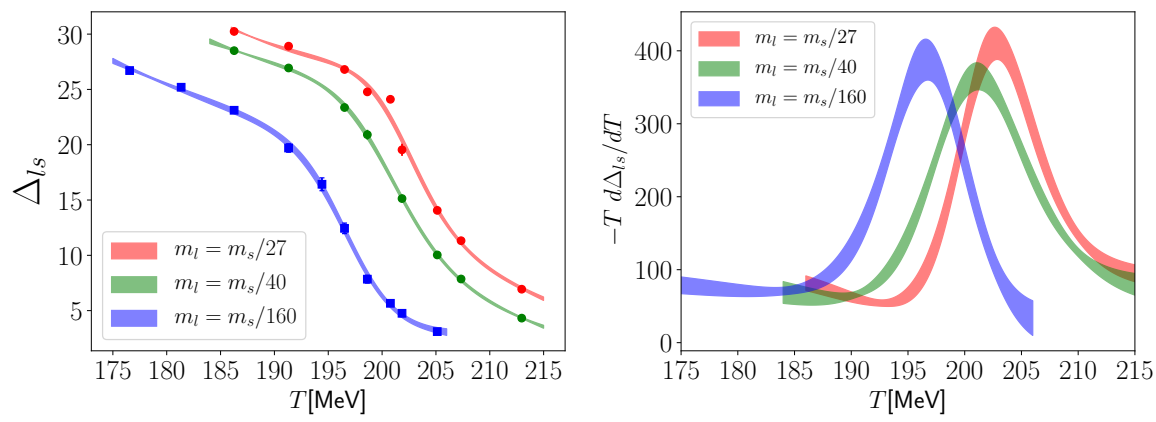

Figure 5: RGI chiral condensate (left) and its temperature derivative (right) versus temperature and for several values of the light quark masses. Shown are results from simulations on lattices with spatial extent $N_{\sigma}=24$. 


\section{Conclusions}

We have presented results from an ongoing study of the phase diagram of (2+1)-flavor QCD in the Roberge-Weiss plane on lattices with temporal extent $N_{\tau}=4$ using the HISQ action with light quark masses decreasing from their physical value towards the chiral limit. Using a finite size scaling analysis we found that the endpoint of the $1^{\text {st }}$ order RW transition line remains $2^{\text {nd }}$ order at least down to light quark masses corresponding to Goldstone pion masses of $55 \mathrm{MeV}$. We have shown that the chiral condensate is sensitive to the RW transition and may serve as a energy-like operator characterizing universal behavior close to the RW endpoint.

\section{Acknowledgement}

This work was supported in part through Contract No. DE-SC001270 with the U.S. Department of Energy, the Deutsche Forschungsgemeinschaft (DFG) through the grant CRC-TR 211 "Strong-interaction matter under extreme conditions" and the grant 05P15PBCAA of the German Bundesministerium für Bildung und Forschung.

\section{References}

[1] R. D. Pisarski and F. Wilczek, Remarks on the Chiral Phase Transition in Chromodynamics, Phys. Rev. D29 (1984) 338.

[2] P. de Forcrand and O. Philipsen, Constraining the QCD phase diagram by tricritical lines at imaginary chemical potential, Phys. Rev. Lett. 105 (2010) 152001 [10 04 . 3144 ].

[3] O. Philipsen and C. Pinke, The $N_{f}=2$ QCD chiral phase transition with Wilson fermions at zero and imaginary chemical potential, Phys. Rev. D93 (2016) 114507 [1602. 0612 9].

[4] C. Czaban, F. Cuteri, O. Philipsen, C. Pinke and A. Sciarra, Roberge-Weiss transition in $N_{f}=2 Q C D$ with Wilson fermions and $N_{\tau}=6$, Phys. Rev. D93 (2016) 054507 [1512.07180].

[5] H. T. Ding, P. Hegde, F. Karsch, A. Lahiri, S. T. Li, S. Mukherjee et al., Chiral phase transition of $(2+1)$-flavor QCD, in 27th International Conference on Ultrarelativistic Nucleus-Nucleus Collisions (Quark Matter 2018) Venice, Italy, May 14-19, 2018, 2018, 1807.05727.

[6] A. Bazavov, H. T. Ding, P. Hegde, F. Karsch, E. Laermann, S. Mukherjee et al., Chiral phase structure of three flavor QCD at vanishing baryon number density, Phys. Rev. D95 (2017) 074505 [1701.03548].

[7] A. Roberge and N. Weiss, Gauge Theories With Imaginary Chemical Potential and the Phases of QCD, Nucl. Phys. B275 (1986) 734.

[8] C. Bonati, M. D’Elia, M. Mariti, M. Mesiti, F. Negro and F. Sanfilippo, Roberge-Weiss endpoint at the physical point of $N_{f}=2+1$ QCD, Phys. Rev. D93 (2016) 074504 [1602.01426].

[9] C. Bonati, E. Calore, M. D’Elia, M. Mesiti, F. Negro, F. Sanfilippo et al., Roberge-Weiss endpoint and chiral symmetry restoration in $N_{f}=2+1 Q C D, 1807.02106$.

[10] L.-K. Wu and F.-L. Zhang, Nature of the chiral phase transition of two flavor QCD from an imaginary chemical potential with HISQ fermions, Phys. Rev. D97 (2018) 114514 [1802 . 00773 ].

[11] K. Rummukainen, M. Tsypin, K. Kajantie, M. Laine and M. E. Shaposhnikov, The Universality class of the electroweak theory, Nucl. Phys. B532 (1998) 283 [hep-lat/9805013].

[12] J. Engels, L. Fromme and M. Seniuch, Numerical equation of state from an improved three-dimensional Ising model, Nucl. Phys. B655 (2003) 277 [cond-mat/0209492]. 